SMART-COP are better at identifying younger, morbidly ill patients with misleadingly low CURB-65 scores requiring early decisions regarding escalation of care.

\section{P196 PREDICTING ESCALATION TO INTENSIVE CARE FOR PATIENTS WITH PNEUMONIA WITH A NEW CLINICAL PREDICTION RULE: SNA 3 P}

${ }^{1} \mathrm{LE}$ Hodgson, ${ }^{1} \mathrm{BD}$ Dimitrov, ${ }^{2} \mathrm{C}$ Stubbs, ${ }^{3} \mathrm{R}$ Venn, ${ }^{4} \mathrm{LG}$ Forni. ${ }^{1}$ University of Southampton, Southampton, UK; ${ }^{2}$ Brighton and Sussex Medical School, Brighton, UK; ${ }^{3}$ Western Sussex Hospitals NHS FT, Worthing, UK; ${ }^{4}$ Royal Surrey County Hospital, Guildford, UK

\subsection{6/thoraxjnl-2016-209333.339}

Introduction Many clinical prediction rules (CPRs) exist for community-acquired pneumonia (CAP), though few have been investigated to predict escalation to an intensive care unit (ICU). Furthermore, most include components (sometimes subjective) that do not allow the potential for electronic automation in clinical practice.

Methods A historical cohort study was performed at two UK adult acute medical units (2013-15). Inclusion was based on an ICD-10 coded diagnosis of pneumonia. Primary outcome was escalation to ICU. Exclusion criteria was: direct ICU admission from $\mathrm{A}$ and $\mathrm{E}$, a stay $<1$ night, age $<18$ or $\geq 80$ or frail elderly, neutropenia, HIV, malignancy and palliative care. Predictive performance of CURB-65 was compared to CRB-65, CURB, the National Early Warning Score (NEWS) and a modified SMARTCOP (SART-CO, as imaging and ABGs were not available) using receiver operating characteristics (ROC) analysis. Multivariable logistic regression was also performed to investigate additional predictors electronically available at admission (blood and physiological parameters).

Results 1,305 of 24,706 medical admissions were included. 8.3\% ( $n=109)$ were escalated to ICU, with significantly increased inpatient mortality $(31 \%$ vs $6.5 \%, \mathrm{p}<0.001)$. 54\% of ICU patients had, or developed, AKI vs $11 \%$ if not escalated $(\mathrm{p}<0.001)$. To predict escalation, AUCROCs for existing CPRs ranged from 0.54-0.61 (Figure). Using multivariable logistic regression a newly derived CPR - SNA ${ }^{3}$ P - (including 6 components: Sodium, NEWS $\geq 7$, Albumin, AST, AKI and Platelets, score range $0-20$ points) demonstrated a statistically significant increase in discrimination (AUCROC 0.80, 95\% CI: 0.75-0.84). At a cut-off of 2 points to predict ICU escalation, sensitivity was 92\% (95\% CI: 85-96\%), specificity 48\% (45-51), positive predictive value (PPV) 14\% (11-16) and negative predictive value $98 \%$ (97-99); at 6 points sensitivity was 53\% (43-63), specificity 87\% (85-89), PPV 27\% (21-33) and NPV 95\% (94-96).

Conclusion Existing pneumonia CPRs, largely derived to predict mortality, have shortcomings when predicting those who require escalation to ICU. The newly derived rule SNA ${ }^{3}$, if externally validated could be incorporated into an electronic clinical decision support system to provide automatic objective assessment and evidence of risk at point-of-care for those who may be considered for escalation.

\section{P197 MEDIUM TERM IMPACTS OF ECMO ON ADULT SURVIVORS}

${ }^{1} \mathrm{MM}$ Abdelaziz, ${ }^{2} \mathrm{~J}$ Fowles, ${ }^{2} \mathrm{~A}$ Vulysteke, ${ }^{2} \mathrm{~K}$ Valchanov, ${ }^{2} \mathrm{~K}$ Sulankey, ${ }^{2} \mathrm{~J}$ Parmar. ${ }^{1}$ University of Cambridge, Cambridge, UK; ${ }^{2}$ Papworth Hospital, Cambridge, UK

10.1136/thoraxjn-2016-209333.340

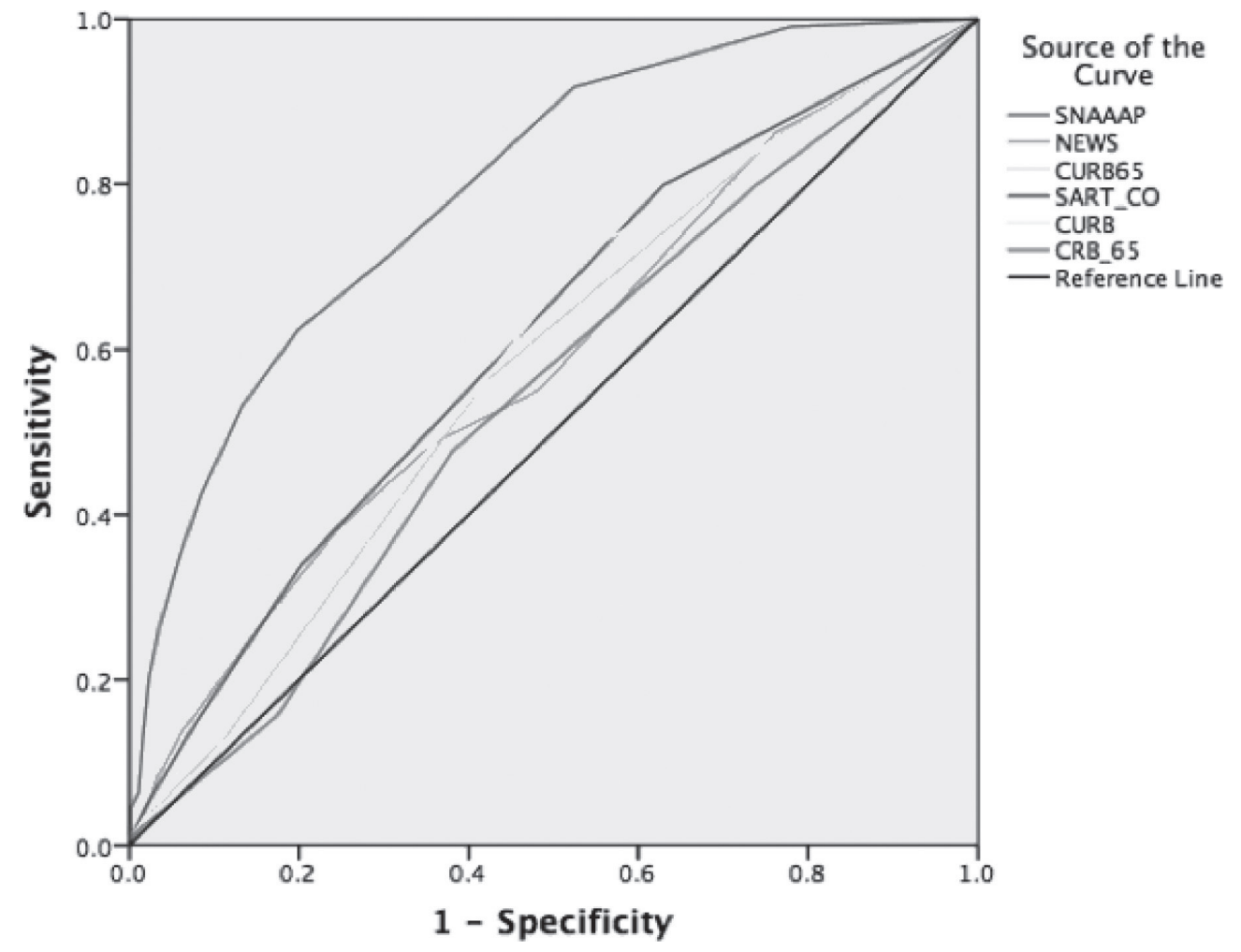

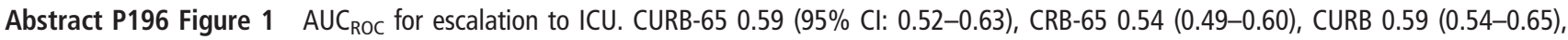
NEWS 0.58 (0.53-0.64), SART-CO $0.61(0.56-0.67)$, SNAAAP $0.80(0.75-0.84)$. 